\title{
Motion Planning of Redundant Manipulators for Painting Uniform Thick Coating in Irregular Duct
}

\author{
Yan Chen, ${ }^{1}$ Wenzhuo Chen, ${ }^{1}$ Ken Chen, ${ }^{2}$ and $\mathrm{Bo} \mathrm{Li}^{1}$ \\ ${ }^{1}$ Department of Petroleum Supply Engineering, Logistical Engineering University, Chongqing 401331, China \\ ${ }^{2}$ Department of Mechanical Engineering, Tsinghua University, Beijing 100084, China \\ Correspondence should be addressed to Yan Chen; yansohucom@sohu.com
}

Received 30 November 2015; Accepted 14 February 2016

Academic Editor: Yangmin Li

Copyright (c) 2016 Yan Chen et al. This is an open access article distributed under the Creative Commons Attribution License, which permits unrestricted use, distribution, and reproduction in any medium, provided the original work is properly cited.

\begin{abstract}
The paper presents a motion planning method of redundant manipulator for painting uniform thick coating on the interior of irregular duct of some aircrafts. Discontinuous peripheral painting method is employed by analyzing the restrictions during painting the duct. For improving the longitudinal uniformity of thick coating, the interlacing painting method plans two sets of sweeping paths and an interlacing distance between the starting paths of the two times of painting. The interlacing distance and overlapping distance are optimized by establishing and analyzing the model of longitudinal uniformity. To enhance the transverse uniformity, sweeping speeds for curved surfaces are calculated by the ratio of transfer efficiency after the basic sweeping speed for the plane is determined. The intertwining method, minimizing the sum of the weighed distances between the duct centerline and key points of the manipulator links, is employed for the joint trajectory planning without collision. The simulation and experiment results show that the redundant manipulators can finish painting the internal surface of the irregular S-shaped duct without collision. The maximum relative deviation is $16.3 \%$ and the thicknesses of all measurement points satisfy the acceptance criteria of the factory.
\end{abstract}

\section{Introduction}

Narrow inlet ducts of some airplanes have S-shaped centerline, whose cross sections transform from circular to irregular quadrangle. Its interior can be seen as a closed surface compounded by several surfaces with different curvatures. It is one of important manufacture processes to paint some inner surfaces of the irregular duct $[1,2]$. Finishing this process by a painting robot can enhance the quality of the coating, improve process speed, reduce pollution, and take workers out of hazardous jobs [3]. Presently, redundant robots are used to paint the interior of the irregular duct by extending a manipulator into it $[4,5]$. The uniformity of thick coating on the duct interior and the collision-free motion of the manipulator are two key aims needed to be achieved by motion planning.

Motion planning including task planning and joint trajectory planning is the base for designing, operating and controlling redundant manipulators for painting the duct interior. Task planning determines the path (including position and orientation) and speed of spray gun during painting tasks. Joint trajectory planning, which determines the time history of position and velocity for each joint, is the key for realizing collision-free motion during painting operation.

There are numerous researchers dealing with task planning problems of painting robot. Their bases for painting task planning are paint deposition models such as Gaussian model [6], parabolic model [7], $\beta$ distribution model [8], and elliptical dual- $\beta$ model [9]. It is hypothesized that coating thickness distribution would satisfy a certain function for establishing these models. Parabolic model and $\beta$ distribution model can well reflect real situation with simple form and thus are more widely used by the researchers. Paint deposition models are fitted by measuring the paint deposition thickness on a plane. Only when the conditions of painting operation accord with or are approximate to the premises of the above models, the task planning will achieve ideal results.

Based on paint deposition model, researchers proposed several methods for path planning of thin coating. Suh et al. [10] developed an automatic trajectory planning system for 
painting robots. They chose the sweeping direction with least curvature. This method would cause worse coating uniformity because of the larger curvature in direction orthogonal to the sweeping direction. Asakawa and Takeuchi [11] developed a motion planning method using the parametric surface to paint a bumper. But how to find the spray overlap distance and the painting velocity was not addressed. The coating thickness was between 13 and $28 \mu \mathrm{m}$, and maximum relative deviation of the thickness was about 58\%. Sheng et al. [12] presented a bounding box method for trajectory generation, which was modified a little by Chen et al. [13]. Its basic concept is that the free surface of the workpiece is divided into many smaller triangles and these generate into some spraying patches with the maximum angular deviation of each area no more than its critical angle, satisfying the requirements of coating thickness deviation. The bounding box method stresses the automation of path planning and the paint coverage on whole surface with little consideration of the optimization of coating uniformity and the influence of shape of complex surfaces. Atkar et al. [14] solved path planning problem on curved surfaces by decomposing it into three subproblems: selecting a seed curve, determining a speed profile along each pass, and selecting the spacing between successive passes. They pointed out that constant sweeping speed caused nonuniform paint deposition near surface boundaries, and in order to solve this problem, they used the paint deposition flux to obtain optimal speed along each pass. But its complex form of the objective function and the presence of the speed and acceleration constraints limit the scope of determining solutions using calculus of variations on a wide class of surfaces, and the experiments showed that the effects of their method on improving coating uniformity seemed not ideal. These researchers only studied the task planning methods for painting once without considering the thick coating which requires multiple times of painting. Therefore, it is desirable to develop an ideal task planning method for uniform thick coating in narrow irregular duct.

Researchers proposed many methods of joint trajectory planning for redundant robots. Potential field is one of the common methods to plan path for redundant manipulators but its biggest problem is that the manipulators can get stuck at local minima. In order to get rid of local minima, search methods are proposed, but when the number of obstacles increases, these methods may cause a manipulator to vibrate between the obstacles [15]. Yoo et al. [16] used a method of computing distance in polyhedron to study motion planning of redundant manipulators. This method is prone to computational complexity, because there would be many points where collision potentially occurs, and these points are subject to change depending on the configuration of the manipulators. Duguleana et al. [17] proposed motion planning of redundant manipulators using neural networks based on reinforcement learning. The principle of neural network method determines that its "solution" can be suboptimal, and even be wrong, but the collision-free problem in the narrow S-shaped duct needs the "solution" to be at least correct. The optimization of the gradient projection method [18] is better than that of the weighted minimum norm solution [19] so that it has been more widely used.

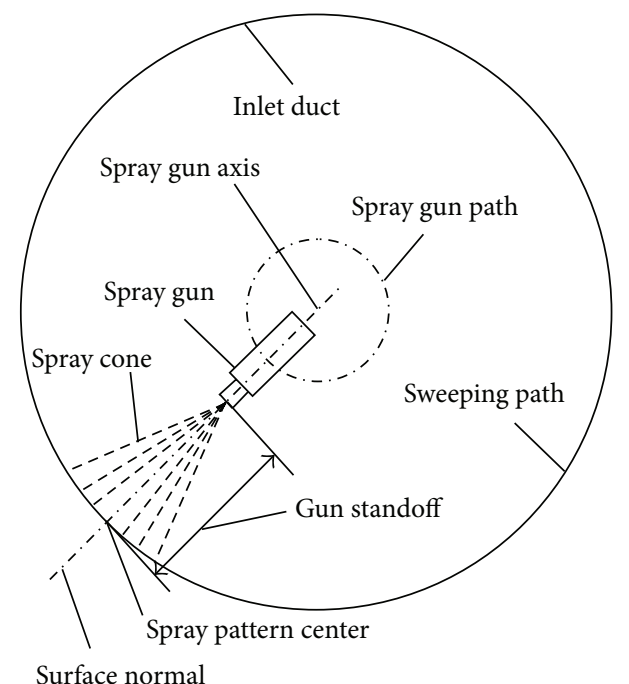

FIGURE 1: Basic principle of painting task planning.

When using the gradient projection method to find inverse kinematic solutions of redundant manipulator, one of the key problems to be solved is to find appropriate optimal functions according to task requirements. In this paper, the intertwining method is proposed to establish the optimal function.

The paper aims to find an appropriate motion planning scheme for redundant painting manipulators to achieve uniform thick coating on the interior of irregular S-shaped duct without collision during operation. It is organized as follows. In Section 2, painting task planning scheme is proposed by analyzing two kinds of uniformities. In Section 3, the intertwining method is proposed to generate the trajectories of robot joint. In Section 4, simulations and experiments are carried out to verify the proposed methods. Section 5 concludes the whole study.

\section{Painting Task Planning}

2.1. Basic Principle of Painting Task Planning. The basic principle of painting task planning is that the axis of spray gun must be perpendicular to the plane of the workpieces or in line with the normal line of the curved surface, with a constant gun standoff. In addition, the paint density must be kept uniform. These principles are important to obtain good surface finish and uniform coating thickness.

Figure 1 shows a sketch of a spray gun painting along a circular path. Painting task planning needs to firstly determine the spray gun orientation, standoff, and sweeping path, and speed and then transfer them to the path and speed of spray gun during painting tasks through coordinate transformation. Sweeping path and speed are defined, respectively, as path and speed of spray pattern center, not those of the spray gun. Gun standoff is determined by the performance of spray gun and paint property, which can be tested through spray experiments. Minimal sweeping speed is restricted by paint property and must insure that the paint would not sag 
on the surface. Maximum speed is confined by paint property and robot performance and must insure that the vibration of the spray gun is acceptable. The basic sweeping speed for the plane can be determined by calculation formula of coating average thickness and painting experiments. Rotation angle of spray gun when painting the interior of a duct must be bigger than that when painting normal workpieces (such as a flat surface) because the painting cross sections are circle, irregular quadrangle, and so on.

2.2. Path Planning of Painting Task. In this part, by analyzing the restrictions during painting the interior of the irregular Sshaped duct, the discontinuous peripheral painting method is proposed. The coating uniformity is divided into transverse uniformity (along the peripheral sweeping path) and longitudinal uniformity (in the direction perpendicular to the peripheral sweeping path). The interlacing method is developed for better longitudinal uniformity, and the ratio of transfer efficiency of two surfaces is used to plan sweeping speed for better transverse uniformity.

2.2.1. Discontinuous Peripheral Painting Method. When painting a curved surface, the sweeping direction of spray cone must be chosen according to the optimal objective. In order to realize uniform coverage, the curvature of the sweeping direction should be the largest, which lessens the influence of curved surface on the adopted paint deposition model, for the spray pattern is usually elliptically shaped. In addition, the paint and the compressed air pipes connected to the spray gun restrict the rotation of the spray gun about the duct centerline to approximately 420 degrees (clockwise or anticlockwise), so that it cannot paint along a thread trajectory which needs rotating arbitrarily. Lastly, the irregular duct cannot rotate during painting process; otherwise, collision would occur. Therefore, the discontinuous peripheral painting method is proposed to paint the interior of the irregular duct in a set of discrete cross sections.

The sweeping paths generated by discontinuous peripheral method, such as A1, A2, and A3 shown in Figure 2, are a series of discrete intersecting lines of the inner surface of the duct and the cross sections that are perpendicular to the duct centerline. The spray pattern center should successively move along these intersecting lines whose shape varies from circle to irregular quadrangle. The transitional paths such as $\mathrm{B} 1$ and $\mathrm{B} 2$ shown in Figure 2 bridge the discrete sweeping paths.

The spray pattern center must move clockwise (or anticlockwise) along a peripheral path such as A1 (Figure 2) during a single painting. Then the spray gun switches off and moves to another peripheral path A2 along a transition line B1. After that, the spray pattern center moves anticlockwise (or clockwise) along the path A2. Other cross sections can be painted in accordance with the above method. The spray gun should switch on and off once for uniform painting when it paints along each intersecting line. In addition, the spray gun enters into the duct and exits from it along the duct centerline.

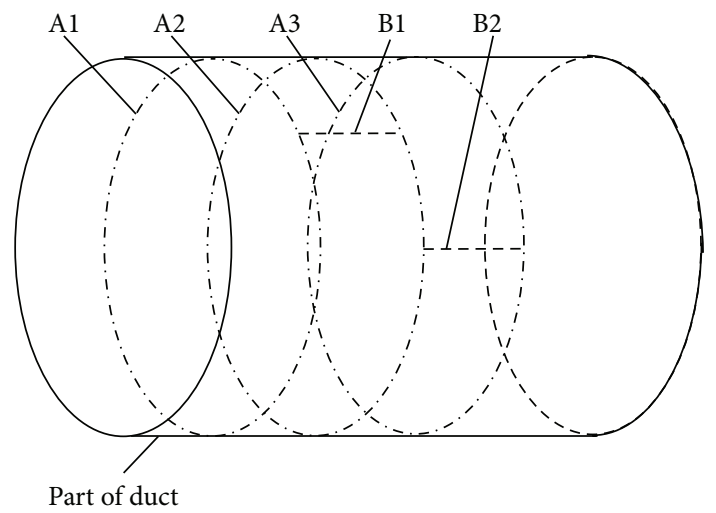

FIGURE 2: Sketch of sweeping path planned by discontinuous peripheral method.

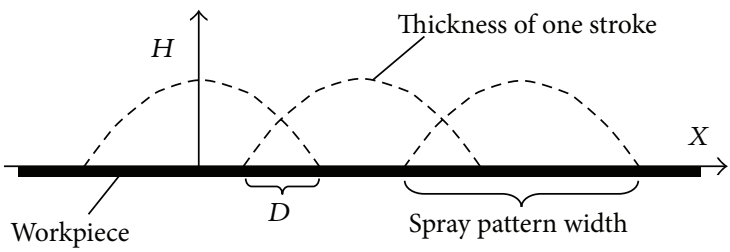

(a) Thickness on every path

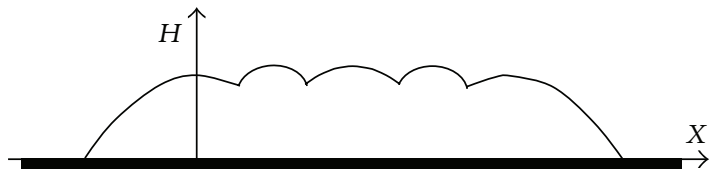

(b) Accumulated thickness distribution

FIGURE 3: Coating thickness distribution of painting once.

2.2.2. Sweeping Path for Interlacing Painting. The thickness distribution of the coating cross section on the plane resulting from painting once will be like what is shown in Figure 3, where $H$ represents the coating thickness, $X$ axis is perpendicular to the direction of sweeping path, and $D$ refers to the overlapping distance. There are periodic crests and troughs in the cross section of the coating. When painting surface with fixed curvature once, if the sweeping direction is along the largest curvature direction, there will also be similar crests and troughs. When painting the interior of the duct, multiple times of painting must be done to achieve the coating with enough thickness. But when planning a set of sweeping paths and painting the plane for multiple times, even with an optimized magnitude of $D$, there exist similar crests and troughs of the coating thickness (Figure 3(b)), and the coating uniformity thus is undesirable. Aiming at this problem, the paper proposes the interlacing painting method to improve the uniformity of the coating by interlacing crests and troughs in two sequential paintings. This method plans two sets of sweeping paths and makes that the position of crests and troughs resulting from one set of sweeping paths appears at the position of troughs and crests resulting from the other. The workpiece is painted along the two sets of paths in turn. 


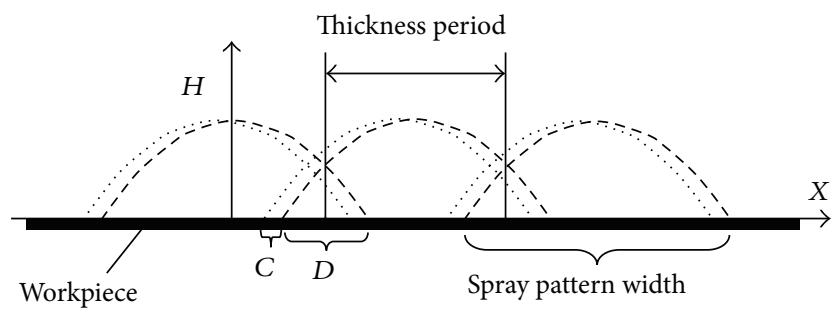

FIGURE 4: Model of interlacing painting.

The model of the interlacing painting is shown as Figure 4, where the heavy line is the cross section of the plane workpiece. The sweeping direction is in line with the cross section normal of the plane workpiece. The broken line and dot line, respectively, represent the coating thickness of every stroke of two times of painting with different sets of sweeping paths. The interlacing painting method adopts two sequential times of painting with the same gun standoff (which makes the same spray pattern width) and the same overlapping distance $D$, but there is an interlacing distance $C$ between the starting paths of the two times of painting.

2.2.3. Dimensionless Interlacing Painting Model. The paint thickness distribution resulting from a single painting stroke can be expressed by parabolic distribution model shown as

$$
H(x)=H_{\max }\left(1-\frac{4 X^{2}}{W^{2}}\right),
$$

where $H(x)$ is the paint thickness distribution of a cross section of the coating, $X$ is the coordinate axis perpendicular to the sweeping paths which are a set of lines, $H_{\max }$ is the maximum thickness, and $W$ is the width of spray pattern.

In this paper, parameters such as $H_{\max }$ and $W$ are determined through experiment and remained constant during painting operation. The parabolic deposition model is nondimensionalized to simplify the calculation process.

Dimensionless parabolic deposition model $h(x)$ can be described as

$$
h(x)=1-4 x^{2}
$$

where $h(x)$ is dimensionless coating thickness of painting once, $h(x)=H(x) / H_{\max }$, and $h(x) \in[0,1] ; x=X / W$ and $x \in[-0.5,0.5]$.

Dimensionless interlacing painting model can be expressed as Figure 5. $d$ represents the dimensionless overlapping distance, $d=D / W$, and $d \in[0,0.5] . c$ is the dimensionless interlacing distance, $c=C / W$, and $c \in[0$, $1-d]$.

2.2.4. Optimized Parameters of Interlacing Painting. Indexes for evaluating the uniformity of coating thickness include relative thickness deviation and thickness variance. Relative thickness deviation is simpler and more explicit, so it is often used as an evaluation method for factories. While thickness variance is more precise and can well reflect the coating

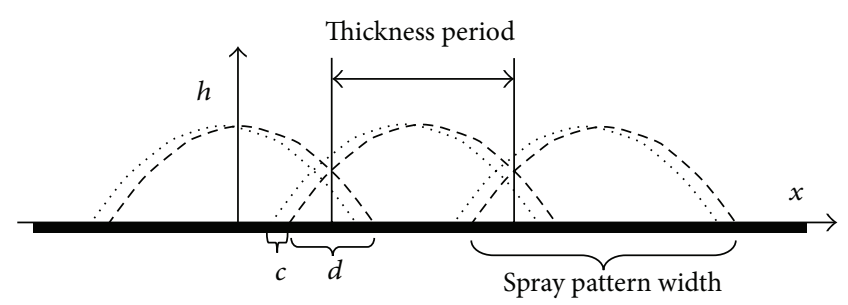

FIGURE 5: Dimensionless interlacing model.

uniformity and thus usually employed as coating uniformity evaluation function in theoretical researches. Therefore, based on thickness variance, longitudinal uniformities of interlacing painting with different parameters ( $c$ and $d$ ) are evaluated. Then the influences of these parameters on the variance of coating thickness are analyzed, and optimized parameters thus are obtained.

Dimensionless longitudinal thickness variance can be described as

$$
v=\frac{1}{l} \int_{l}(f-\bar{f})^{2} d l
$$

where $f$ is the dimensionless coating thickness at a point, $l$ represents the dimensionless width of the workpiece, and $\bar{f}$ is the dimensionless mean coating thickness.

The average film thickness $\bar{h}$ of interlacing painting in one coating thickness period is

$$
\begin{aligned}
\bar{h} & =\frac{4}{1-d}\left\{\int_{0}^{0.5-d} h(x) d x\right. \\
& \left.+\int_{0.5-d}^{0.5-0.5 d}[h(x)+h(x-1+d)] d x\right\} .
\end{aligned}
$$

When $c \leq 0.5 d$ and $c+d \leq 0.5$, shown in Figure 5,

$$
\begin{aligned}
v= & \frac{1}{1-d}\left\{\int_{0.5 d-0.5}^{d-0.5-c}[h(x)+h(x+c)+h(x+1-d)\right. \\
& +h(x+1-d+c)-\bar{h}]^{2} d x+\int_{d-0.5-c}^{d-0.5}[h(x) \\
& +h(x+c)+h(x+1-d)-\bar{h}]^{2} d x \\
& +\int_{d-0.5}^{0.5-d-c}[h(x)+h(x+c)-\bar{h}]^{2} d x \\
& +\int_{0.5-d-c}^{0.5-d}[h(x)+h(x+c)+h(x-1+d+c) \\
& -\bar{h}]^{2} d x+\int_{0.5-d}^{0.5-0.5 d}[h(x)+h(x+c) \\
& \left.+h(x-1+d+c)+h(x-1+d)-\bar{h}]^{2} d x\right\} .
\end{aligned}
$$




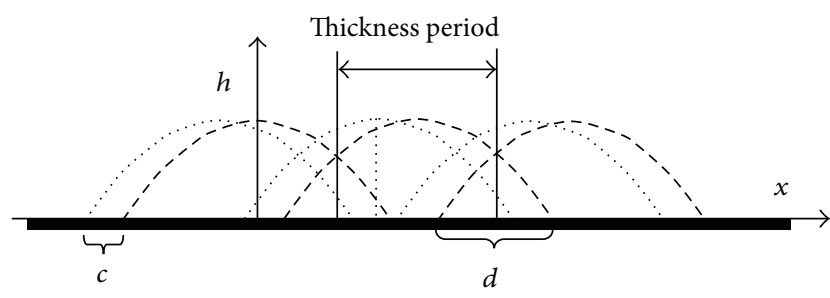

Figure 6: Coating thickness distribution when $c \leq 0.5 d, c+d>0.5$ and $c+2 d \leq 1$.

When $c \leq 0.5 d, c+d>0.5$ and $c+2 d \leq 1$, shown in Figure 6,

$$
\begin{aligned}
v & =\frac{1}{1-d}\left\{\int_{0.5 d-0.5}^{d-0.5-c}[h(x)+h(x+c)+h(x+1-d)\right. \\
& +h(x+1-d+c)-\bar{h}]^{2} d x+\int_{d-0.5-c}^{d-0.5}[h(x) \\
& +h(x+c)+h(x+1-d)-\bar{h}]^{2} d x \\
& +\int_{d-0.5}^{0.5-d-c}[h(x)+h(x+c)-\bar{h}]^{2} d x \\
& +\int_{0.5-d-c}^{0.5-d}[h(x)+h(x+c)+h(x-1+d+c) \\
& -\bar{h}]^{2} d x+\int_{0.5-d}^{0.5-0.5 d}[h(x)+h(x+c) \\
& \left.+h(x-1+d+c)+h(x-1+d)-\bar{h}]^{2} d x\right\} .
\end{aligned}
$$

When $c \leq 0.5 d, c+d>0.5$ and $c+2 d>1$, shown in Figure 7 ,

$$
\begin{aligned}
v & =\frac{1}{1-d}\left\{\int_{0.5 d-0.5}^{d-0.5-c}[h(x)+h(x+c)+h(x+1-d)\right. \\
& +h(x+1-d+c)-\bar{h}]^{2} d x+\int_{d-0.5-c}^{0.5-d-c}[h(x) \\
& +h(x+c)+h(x+1-d)-\bar{h}]^{2} d x \\
& +\int_{0.5-d-c}^{d-0.5}[h(x)+h(x+c)+h(x+1-d)+h(x \\
& -1+d+c)-\bar{h}]^{2} d x+\int_{d-0.5}^{0.5-d}[h(x)+h(x \\
& +c)+h(x-1+d+c)-\bar{h}]^{2} d x \\
& +\int_{0.5-d}^{0.5-0.5 d}[h(x)+h(x+c)+h(x-1+d+c) \\
& \left.+h(x-1+d)-\bar{h}]^{2} d x\right\} .
\end{aligned}
$$

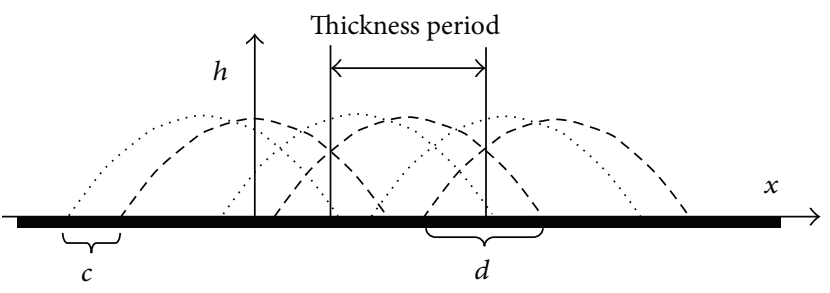

FIGURE 7: Coating thickness distribution when $c \leq 0.5 d, c+d>0.5$ and $c+2 d>1$.

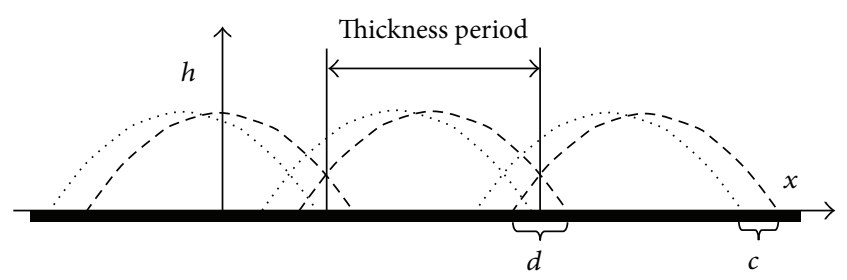

FIGURE 8: Coating thickness distribution when $0.5 d<c \leq d$ and $c+d \leq 0.5$.

When $0.5 d<c \leq d$ and $c+d \leq 0.5$, shown in Figure 8,

$$
\begin{aligned}
v & =\frac{1}{1-d}\left\{\int_{0.5 d-0.5}^{d-0.5}[h(x)+h(x+c)+h(x+1-d)\right. \\
& -\bar{h}]^{2} d x+\int_{d-0.5}^{0.5-d-c}[h(x)+h(x+c) \\
& -\bar{h}]^{2} d x+\int_{0.5-d-c}^{0.5-d}[h(x)+h(x+c)+h(x \\
& -1+d+c)-\bar{h}]^{2} d x+\int_{0.5-d}^{0.5-c}[h(x)+h(x \\
& +c)+h(x-1+d+c)+h(x-1+d)-\bar{h}]^{2} d x \\
& +\int_{0.5-c}^{0.5-0.5 d}[h(x)+h(x-1+d+c) \\
& \left.+h(x-1+d)-\bar{h}]^{2} d x\right\} .
\end{aligned}
$$

When $0.5 d<c \leq d, c+d>0.5$ and $c+2 d \leq 1$, shown in Figure 9,

$$
\begin{aligned}
v & =\frac{1}{1-d}\left\{\int_{0.5 d-0.5}^{d-0.5}[h(x)+h(x+c)+h(x+1-d)\right. \\
& -\bar{h}]^{2} d x+\int_{d-0.5}^{0.5-d-c}[h(x)+h(x+c) \\
& -\bar{h}]^{2} d x+\int_{0.5-d-c}^{0.5-d}[h(x)+h(x+c)+h(x \\
& -1+d+c)-\bar{h}]^{2} d x+\int_{0.5-d}^{0.5-c}[h(x)+h(x \\
& +c)+h(x-1+d+c)+h(x-1+d)-\bar{h}]^{2} d x
\end{aligned}
$$




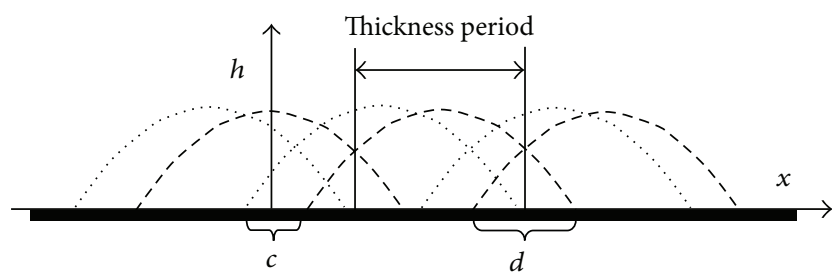

FIGURE 9: Coating thickness distribution when $0.5 d\langle c \leq d, c+d\rangle$ 0.5 and $c+2 d \leq 1$.

$$
\begin{aligned}
& +\int_{0.5-c}^{0.5-0.5 d}[h(x)+h(x-1+d+c) \\
& \left.+h(x-1+d)-\bar{h}]^{2} d x\right\} .
\end{aligned}
$$

When $0.5 d<c \leq d, c+d>0.5, c+2 d>1$ and $c+1.5 d \leq 1$, shown in Figure 10,

$$
\begin{aligned}
v= & \frac{1}{1-d}\left\{\int_{0.5 d-0.5}^{0.5-d-c}[h(x)+h(x+c)+h(x+1-d)\right. \\
& -\bar{h}]^{2} d x+\int_{0.5-d-c}^{d-0.5}[h(x)+h(x+c)+h(x \\
& -1+d+c)+h(x+1-d)-\bar{h}]^{2} d x
\end{aligned}
$$

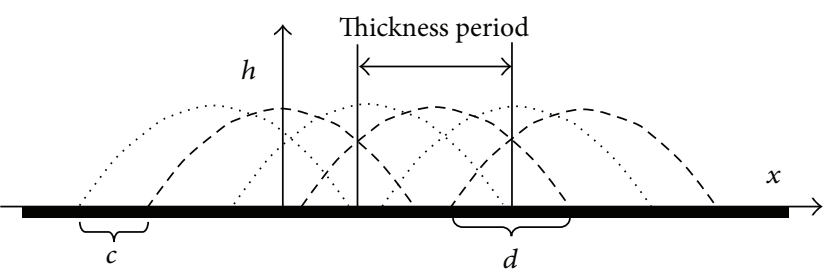

FIGURE 10: Painting when $0.5 d<c \leq d, c+d>0.5, c+2 d>1$ and $c+1.5 d \leq 1$.

$$
\begin{aligned}
& +\int_{d-0.5}^{0.5-d}[h(x)+h(x+c)+h(x-1+d+c) \\
& -\bar{h}]^{2} d x+\int_{0.5-d}^{0.5-c}[h(x)+h(x+c)+h(x \\
& -1+d+c)+h(x-1+d)-\bar{h}]^{2} d x \\
& +\int_{0.5-c}^{0.5-0.5 d}[h(x)+h(x-1+d+c) \\
& \left.+h(x-1+d)-\bar{h}]^{2} d x\right\} .
\end{aligned}
$$

When $0.5 d<c \leq d, c+d>0.5, c+2 d>1$ and $c+1.5 d>1$, shown in Figure 11,

$$
\begin{aligned}
v & =\frac{1}{1-d}\left\{\int_{0.5 d-0.5}^{d-0.5}[h(x)+h(x+c)+h(x+1-d)+h(x-1+d+c)-\bar{h}]^{2} d x\right. \\
& +\int_{d-0.5}^{0.5-d}[h(x)+h(x+c)+h(x-1+d+c)-\bar{h}]^{2} d x \\
& +\int_{0.5-d}^{0.5-c}[h(x)+h(x+c)+h(x-1+d+c)+h(x-1+d)-\bar{h}]^{2} d x \\
& +\int_{0.5-c}^{1.5-2 d-c}[h(x)+h(x-1+d+c)+h(x-1+d)-\bar{h}]^{2} d x \\
& \left.+\int_{1.5-2 d-c}^{0.5-0.5 d}[h(x)+h(x-1+d+c)+h(x-1+d)+h(x-2+c+2 d)-\bar{h}]^{2} d x\right\} .
\end{aligned}
$$

When $c>d$ and $c+d \leq 0.5$, shown in Figure 12,

$$
\begin{aligned}
v & =\frac{1}{1-d}\left\{\int_{0.5 d-0.5}^{d-0.5}[h(x)+h(x+c)+h(x+1-d)\right. \\
& -\bar{h}]^{2} d x+\int_{d-0.5}^{0.5-d-c}[h(x)+h(x+c) \\
& -\bar{h}]^{2} d x+\int_{0.5-d-c}^{0.5-c}[h(x)+h(x+c)+h(x \\
& -1+d+c)-\bar{h}]^{2} d x+\int_{0.5-c}^{0.5-d}[h(x)+h(x
\end{aligned}
$$

$$
\begin{aligned}
& -1+d+c)-\bar{h}]^{2} d x+\int_{0.5-d}^{0.5-0.5 d}[h(x) \\
& \left.+h(x-1+d+c)+h(x-1+d)-\bar{h}]^{2} d x\right\} .
\end{aligned}
$$

When $c>d, c+d>0.5$ and $c+2 d \leq 1$, shown in Figure 13,

$$
\begin{aligned}
v & =\frac{1}{1-d}\left\{\int_{0.5 d-0.5}^{d-0.5}[h(x)+h(x+c)+h(x+1-d)\right. \\
& -\bar{h}]^{2} d x+\int_{d-0.5}^{0.5-d-c}[h(x)+h(x+c)
\end{aligned}
$$




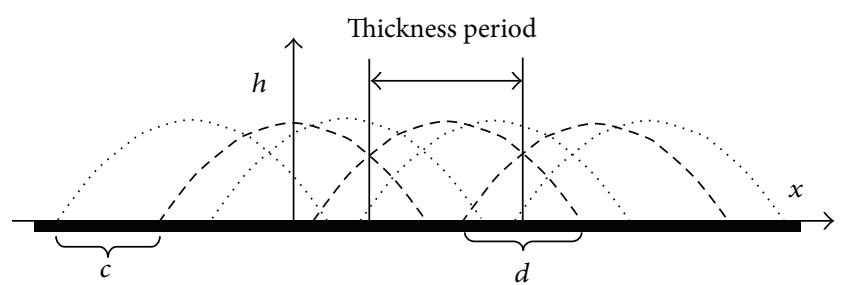

FIGURE 11: Painting when $0.5 d<c \leq d, c+d>0.5, c+2 d>1$ and $c+1.5 d>1$.

$$
\begin{aligned}
& -\bar{h}]^{2} d x+\int_{0.5-d-c}^{0.5-c}[h(x)+h(x+c)+h(x \\
& -1+d+c)-\bar{h}]^{2} d x+\int_{0.5-c}^{0.5-d}[h(x)+h(x \\
& -1+d+c)-\bar{h}]^{2} d x+\int_{0.5-d}^{0.5-0.5 d}[h(x) \\
& \left.+h(x-1+d+c)+h(x-1+d)-\bar{h}]^{2} d x\right\} .
\end{aligned}
$$

When $c>d, c+d>0.5$ and $c+1.5 d \leq 1$, shown in Figure 14,

$$
\begin{aligned}
v= & \frac{1}{1-d}\left\{\int_{0.5 d-0.5}^{0.5-c-d}[h(x)+h(x+c)+h(x+1-d)\right. \\
& -\bar{h}]^{2} d x+\int_{0.5-d-c}^{d-0.5}[h(x)+h(x+1-d) \\
& +h(x-1+c+d)+h(x+c)-\bar{h}]^{2} d x \\
& +\int_{d-0.5}^{0.5-c}[h(x)+h(x-1+c+d)+h(x+c) \\
& -\bar{h}]^{2} d x+\int_{0.5-c}^{0.5-d}[h(x)+h(x-1+d+c) \\
& -\bar{h}]^{2} d x+\int_{0.5-d}^{0.5-0.5 d}[h(x) \\
& \left.+h(x-1+d+c)+h(x-1+d)-\bar{h}]^{2} d x\right\} .
\end{aligned}
$$

When $c>d, c+d>0.5$ and $c+1.5 d>1$, shown in Figure 15,

$$
\begin{aligned}
v= & \frac{1}{1-d}\left\{\left\{\int_{0.5 d-0.5}^{d-0.5}[h(x)+h(x+c)+h(x+1-d)+h(x-1+d+c)-\bar{h}]^{2} d x\right.\right. \\
& +\int_{d-0.5}^{0.5-c}[h(x)+h(x+c)+h(x-1+d+c)-\bar{h}]^{2} d x+\int_{0.5-c}^{0.5-d}[h(x)+h(x-1+d+c)-\bar{h}]^{2} d x \\
& +\int_{0.5-d}^{1.5-2 d-c}[h(x)+h(x-1+d+c)+h(x-1+d)-\bar{h}]^{2} d x \\
& \left.+\int_{1.5-2 d-c}^{0.5-0.5 d}[h(x)+h(x-1+d+c)+h(x-1+d)+h(x-2+c+2 d)-\bar{h}]^{2} d x\right\}
\end{aligned}
$$

$v$ is such a complicated function of $c$ and $d$ that it is difficult to perceive their relation. Their relation is shown in Figure 16 by numerical calculation through (4) to (15). The value of $v$ is smaller in 4 areas: when $c \in[0.495(1-$ $d), 0.505(1-d)]$ and $d \in[0.1800,0.1925], v$ is around 0.0009 ; when $c \in[0.220(1-d), 0.270(1-d)]$ and $d \in[0.2950,0.3075]$, $v \in[0.0007,0.0009]$; when $c \in[0.730(1-d), 0.780(1-$ $d)]$ and $d \in[0.2950,0.3075], v \in[0.0007,0.0009]$; when $c \in[0.475(1-d), 0.525(1-d)]$ and $d \in[0.4100,0.4375]$, $v \in[0.0005,0.0009]$. Better thickness uniformity can be realized theoretically by choosing path parameters within these areas.

From the analysis of Figure 16, $c$ and $d$ are key influencing factors of longitudinal thickness uniformity. There are three advantages if $d$ is set around 0.3 during painting process. It can lessen the influence of starting path on coating uniformity, the influence of path deviation on coating uniformity, and the bad effect on coating uniformity of odd number of painting times. Therefore, the area of $c \in[0.220(1-$ $d), 0.270(1-d)]$ and $d \in[0.2950,0.3075]$ or the area of $c \in[0.730(1-d), 0.780(1-d)]$ and $d \in[0.2950,0.3075]$ should be chosen. The sweeping paths of the two areas are same in essence.

2.3. Planning Painting Speed. In this part, the transverse uniformity is considered to plan sweeping speed. And this uniformity is achieved through keeping the average coating thickness $\bar{H}$ unchanged along a sweeping path.

Suppose that one-stroke painting is done on a surface with constant curvature with the painting flux $Q$ at the sweeping speed $V$. The stroke length is $L$. The spray pattern width is $W$. The transfer efficiency is $\eta$, which is the percentage of total coating solids employed by a coating applicator which adheres to the object being coated. During the period of $t$, the average coating thickness on the surface $\bar{H}$ can be expressed as

$$
\bar{H}=\frac{Q \zeta(\eta t)}{W L} .
$$




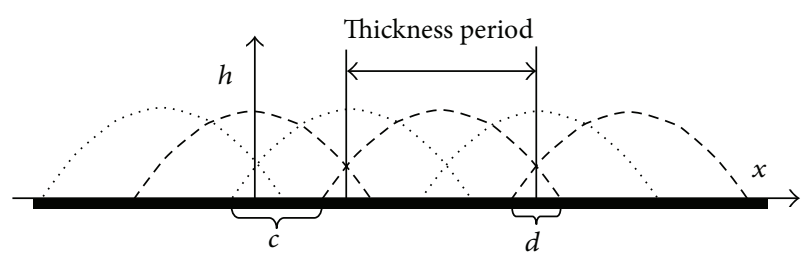

FIGURE 12: Coating thickness distribution when $c>d$ and $c+d \leq$ 0.5 .

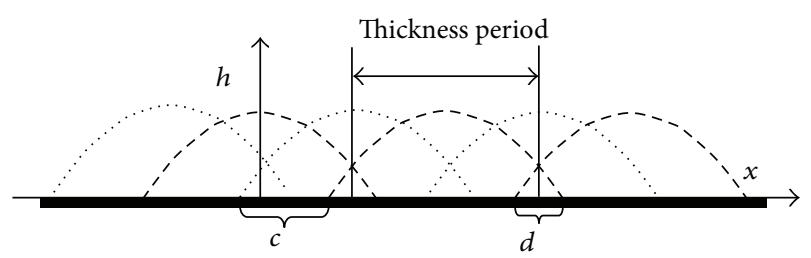

FIGURE 13: Coating thickness distribution when $c>d, c+d>0.5$ and $c+2 d \leq 1$.

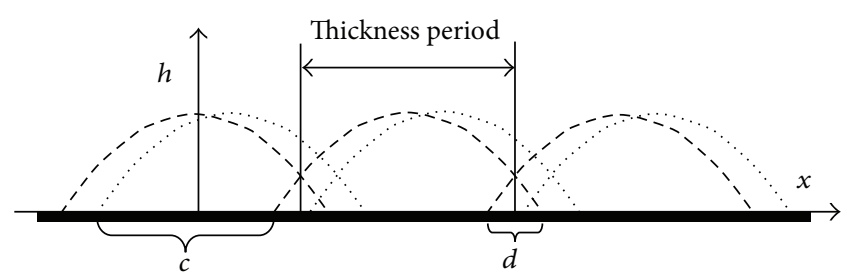

FIGURE 14: Coating thickness distribution when $c>d, c+d>0.5$ and $c+1.5 d \leq 1$.

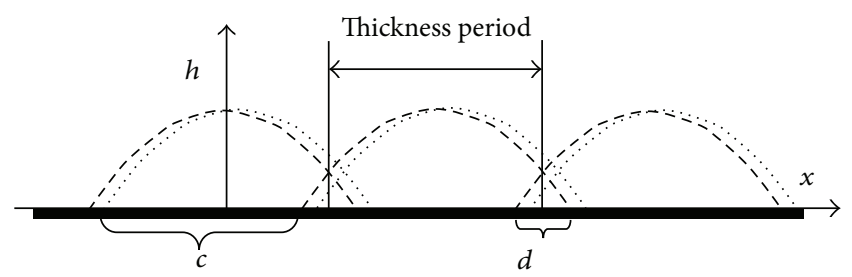

FIGURE 15: Coating thickness distribution when $c>d, c+d>0.5$ and $c+1.5 d>1$.

By substituting $V=L / t$ in to (16), we obtain

$$
\bar{H}=\frac{Q \zeta \eta}{W V} .
$$

It can be seen that, according to (17), the average coating thickness on the workpiece is proportional to the transfer efficiency $\eta$ and inversely proportional to sweeping speed $V$. Because the painting process adopts constant painting flux and unchanged gun standoff, the volume fraction of solid constituent $\zeta$ and the spray pattern width $W$ can be seen as invariable. Therefore, the average coating thickness $\bar{H}$ is only related to the values of $\eta$ and $V$. In order to find a way to maintain constant $\bar{H}$ on a surface with changing curvature, the factors affecting the value of $\eta$ should be found.

Transfer efficiency $\eta$ is mainly influenced by the painting angle, gun standoff, sweeping speed, and shape (especially

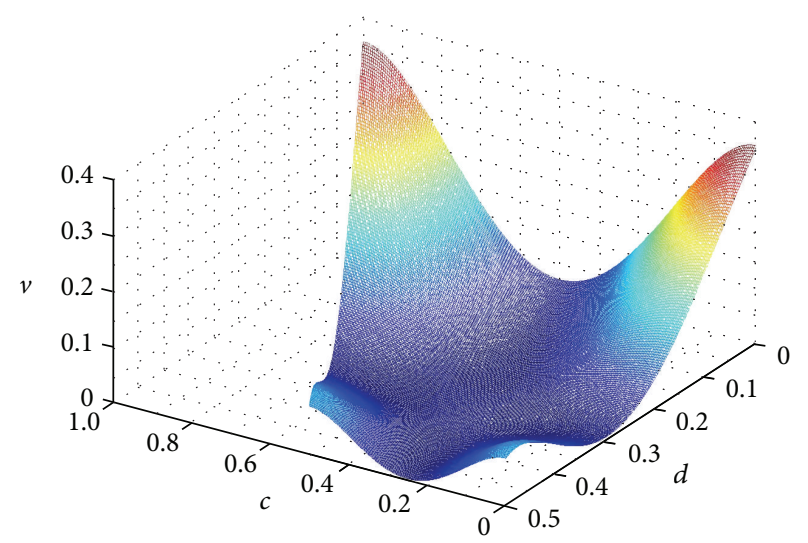

FIGURE 16: Relationship of $v$ with $d$ and $c$.

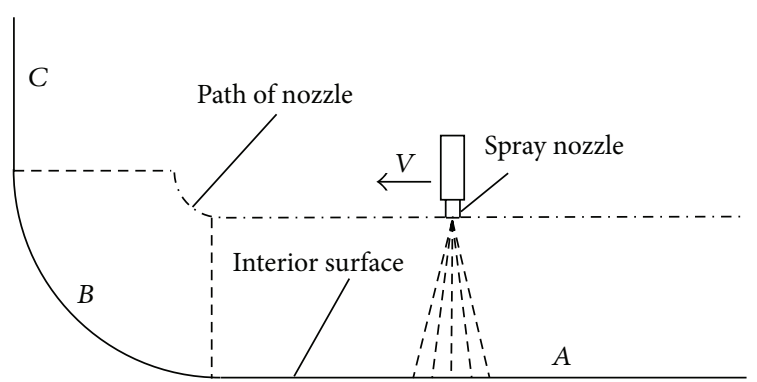

FIGURE 17: Typical sketch of peripheral painting path.

curvature in our research) of workpiece. The painting angle and gun standoff are constant in this research, so they can be neglected. The influence of minor changes of the sweeping speed on $\eta$ can be neglected, because the sweeping speed is usually no more than $20 \mathrm{~cm} / \mathrm{s}$ when painting the duct and is much lower than the speed of compressed air and paint particulate (close to the sound speed). Therefore, it is enough only to consider the influence of the curvature on the magnitude of $\eta$. The inner surface with bigger curvature makes greater transfer efficiency.

Figure 17 shows a typical sketch of a spray gun painting along a peripheral path. If the coating thicknesses on sections $A$ and $B$ are equal, the following equations can be obtained.

$$
\begin{aligned}
\frac{Q \zeta \eta_{A}}{W V_{A}} & =\frac{Q \zeta \eta_{B}}{W V_{B}}, \\
\frac{\eta_{A}}{V_{A}} & =\frac{\eta_{B}}{V_{B}} .
\end{aligned}
$$

Assume that the curvature of section $B$ is greater than that of section $A$, then $\eta_{B}$ is greater than $\eta_{A}$. Define the ratio of transfer efficiency of two surfaces:

$$
\beta=\frac{\eta_{B}}{\eta_{A}} .
$$

Combining (19) and (20), we obtain

$$
V_{B}=\beta V_{A} .
$$


The ratio of transfer efficiency can be obtained by experiments or simulation of computational fluid dynamics. After the basic sweeping speed for the plane is determined, the sweeping speeds of curved surfaces can be calculated.

\section{Joint Trajectory Planning}

Joint trajectory refers to the position, velocity, and acceleration for each DOF of manipulators at run time. Its planning is the bases for the operation and control of robot.

Coordinate systems from $\{0\}$ to $\{i\}$ are attached, respectively, on redundant painting manipulators with $i$ joints by using D-H approach. Then, the description of the position and orientation of spray gun in the coordinate system $\{0\},{ }_{p}^{0} \mathbf{T}$, can be obtained as

$$
{ }_{p}^{0} \mathbf{T}={ }_{1}^{0} \mathbf{T}_{2}^{1} \mathbf{T}_{3}^{2} \mathbf{T}_{4}^{3} \mathbf{T}_{5}^{4} \mathbf{T}_{6}^{5} \mathbf{T} \cdots{ }_{i}^{i-1} \mathbf{T}{ }_{p}^{i} \mathbf{T},
$$

where ${ }_{i}^{i-1} \mathbf{T}$ is the position and orientation of the coordinate system $\{i\}$ in the coordinate system $\{i-1\}$, and ${ }_{p}^{i} \mathbf{T}$ is the position and orientation of spray gun in the coordinate system $\{i\}$.

The differential relation between Cartesian velocities of the redundant manipulators and the joint velocities is

$$
\dot{\mathbf{X}}=\mathbf{J} \dot{\mathbf{q}}
$$

where $\dot{\mathbf{X}}$ is the vector of translational and rotational velocities of the spray gun with reference to base coordinates, $\dot{\mathbf{q}}$ is the vector of $n$ joint rates, and $\mathbf{J}$ is the Jacobian matrix ( $m$ by $n$, $m<n) . m$ denotes the space DOF.

The gradient projection method is used to solve (23), and the result can be expressed as

$$
\dot{\mathbf{q}}=\mathbf{J}^{+} \dot{\mathbf{X}}+\left(\mathbf{I}-\mathbf{J}^{+} \mathbf{J}\right) \mathbf{E},
$$

where $\mathbf{J}^{+}=\mathbf{J}^{T}\left(\mathbf{J J}^{T}\right)^{-1}$ is the Moore-Penrose pseudoinverse of $\mathbf{J}, \mathbf{I}$ is the unit matrix $(n \times n)$, and $\mathbf{E}=-\lambda \nabla F(\mathbf{q}) \cdot \lambda$ is a real scalar constant coefficient, and $\nabla F(\mathbf{q})$ is the gradient vector of $F(\mathbf{q})$ which is the objective function to be optimized.

As the number of the solution of (24) is infinite, some restrictions are needed to obtain a unique solution which is optimistic. The intertwining method is proposed to obtain a unique solution of (24): the distance between the centerline and each part of the manipulators will be controlled so that the manipulators can intertwine the duct centerline closely without collision in the duct. The distance between the inner surface of the duct and each part of the manipulators should be limited to ensure collision-free operation. It is hard to calculate the distance due to the complexity of the duct. But it is much simpler to calculate the distance between the duct centerline and each part of the manipulators. And controlling the distance between each part of the manipulators and the centerline is equivalent to controlling the distance between the inner surface and the parts. If the manipulators intertwine the duct centerline closely, they would work without collision in the duct. Therefore, it can build the collision-free restriction to calculate the distance sum of the duct centerline and all joints of the manipulators. The midpoint and ends of each link are defined to be key points. If these points were all near to the duct centerline, there would be no collision. The weighed distance sum of the duct centerline and the link key points, $G$, can be calculated as

$$
\begin{aligned}
G & =\sum_{i=1}^{\alpha}\left[k_{x}\left(x_{i}-x_{\mathrm{O} i}\right)^{2}+k_{y}\left(y_{i}-y_{\mathrm{O} i}\right)^{2}\right. \\
& \left.+k_{z}\left(z_{i}-z_{\mathrm{O} i}\right)^{2}\right],
\end{aligned}
$$

where $x_{i}, y_{i}$, and $z_{i}$ are the coordinate of the link key point $i$ in the base, $x_{\mathrm{O} i}, y_{\mathrm{O} i}$, and $z_{\mathrm{O} i}$ are the coordinate of the center point of the duct cross section, $k_{x}, k_{y}$, and $k_{z}$ are the weighed coefficient in the direction of $x, y$, and $z$, and $\alpha$ is the key point number.

Then, an objective function $F$ can be obtained by minimizing the weighed distance sum of the duct centerline and the link key points:

$$
F=\min (G) .
$$

After (25) and (26) are substituted into (24), we obtain

$$
\begin{aligned}
\dot{\mathbf{q}} & =\mathbf{J}^{+} \dot{\mathbf{X}}-\lambda\left(\mathbf{I}-\mathbf{J}^{+} \mathbf{J}\right) \nabla \sum_{i=1}^{\alpha}\left[k_{x}\left(x_{i}-x_{\mathrm{O} i}\right)^{2}\right. \\
& \left.+k_{y}\left(y_{i}-y_{\mathrm{O} i}\right)^{2}+k_{z}\left(z_{i}-z_{\mathrm{O} i}\right)^{2}\right] .
\end{aligned}
$$

By solving (27), optimized joint trajectories can be obtained.

Before task planning, preprocessing should be set up. The trajectory of the spray nozzle and the duct center line should be calculated. Suppose that the path points are $\mathbf{x}_{i}^{d}(i=$ $0,1,2, \ldots, n)$ and that the forward kinematics is $\mathbf{X}_{0}=f\left(\mathbf{q}_{0}\right)$. Iteration step size $\Delta t$ and iteration error should be set, and initial index $i$ should be set as 0 . Then, the process of joint trajectory planning is as follows.

Step 1. Calculate the velocity of the nozzle in task space by using difference quotient of two consecutive path points, which is $\dot{\mathbf{x}}=\left(\mathbf{x}_{i+1}^{d}-\mathbf{x}_{i}^{d}\right) / \Delta t$.

Step 2. Set the weighed coefficient $\lambda$ and real scalar constant coefficient $k$.

Step 3. In the current configuration $\mathbf{q}_{i}$, calculate the Jacobian matrix, its pseudo inverse $\mathbf{J}^{\dagger}=\mathbf{J}^{T}\left(\mathbf{J J}^{T}\right)^{-1}$, and the optimized vector $E$.

Step 4. Compute the joint velocity $\dot{\mathbf{q}}$ by using (25) and the current configuration $\mathbf{q}_{i}^{\prime}$, where $\mathbf{q}_{i}^{\prime}=\mathbf{q}_{i}+\Delta t \cdot \dot{\mathbf{q}}$.

Step 5. Calculate position and orientation $\mathbf{x}$ of the spray nozzle with robot configuration as $\mathbf{q}_{i}^{\prime}$, where $\mathbf{x}=f\left(\mathbf{q}_{i}^{\prime}\right)$; calculate the error vector $\boldsymbol{\xi}$, where $\boldsymbol{\xi}=\mathbf{x}_{i+1}^{d}-\mathbf{x}$. If $\|\boldsymbol{\xi}\|<\varepsilon$, the iteration will break and $\mathbf{q}_{i}=\mathbf{q}_{i}^{\prime}$; if $\|\boldsymbol{\xi}\|>\varepsilon$, let $\mathbf{q}_{i}=\mathbf{q}_{i}^{\prime}$ and $\dot{\mathbf{x}}=\boldsymbol{\xi} / \Delta t$, and then return to Step 3 .

Step 6. Check if there is collision during motion. If collision exists, return to Step 2; If not, let $i=i+1$ and return back to Step 1 , until $i=n$. 


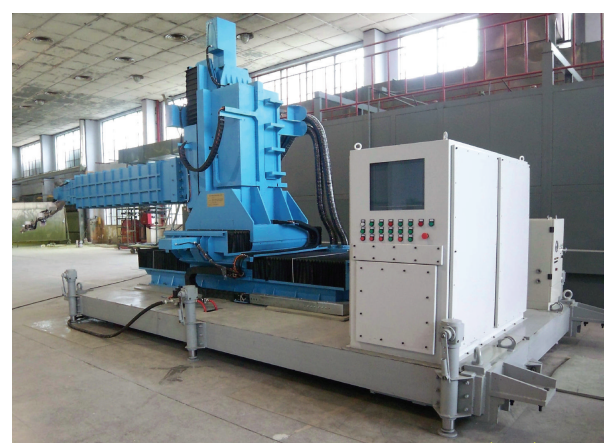

FIGURE 18: Painting robot.

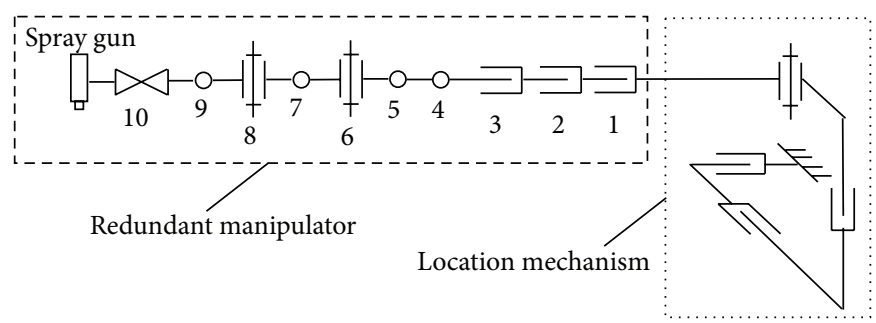

FIGURE 19: Robot mechanism sketch.

Step 7. After obtaining the path points in joint space, calculate the path $\{\mathbf{q}(t), \dot{\mathbf{q}}(t), \ddot{\mathbf{q}}(t)\}$ by using third-order polynomial.

\section{Simulation and Experiment}

The simulation and experiment adopted a painting robot with a 10-DOF manipulator for painting uniform thick coating in an irregular S-shaped duct. The robot is shown as Figure 18 and its mechanism sketch is shown as Figure 19. The 10-DOF manipulator consists of three prismatic joints (joints 1,2 , and 3 ) and seven revolute joints (joints 4-10). The axes of joints 1 , 2 , and 3 are in the same direction. The axes of joints 6,8 , and 10 are perpendicular to the axes of joints 4, 5, 7, and 9. Joint 10 is designed as the last joint of the painting manipulator linking the spray gun.

The motion planning method proposed above was implemented so that the painting path and joint trajectory were generated, and the motion of the robot was firstly simulated in the software. The 3D models of the painting manipulator and the duct were also built according to their real size (Figure 20) by Pro/E. The generated trajectory data were transferred into the Pro/E to check if there would be any motion interference during painting operation. The result of simulation indicated that no collision would occur. Then, during the operation of the painting manipulator in the duct, the minimal distance between the duct and the manipulators was $18 \mathrm{~mm}$, which satisfied the safety requirement.

Three typical parts including the bottom of the front, the top of the front, and the back of the irregular S-shaped duct were chosen to be painted and no collision occurred. The painting experiment was conducted as shown in Figure 21. The pressure for both spray pattern and the atomization was $0.28 \mathrm{MPa}$. The parameters of dynamic surge suppressor

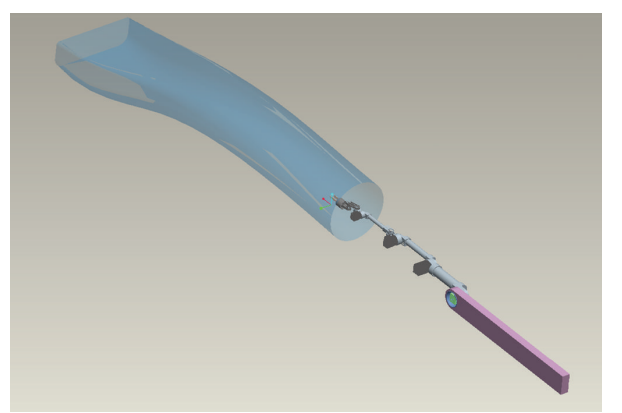

FIgURE 20: Simulation model for painting motion in Pro/E.

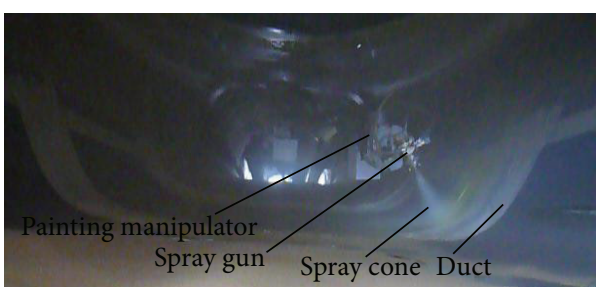

FIgURE 21: Painting experiment in irregular duct.

and the paint agitator were set as $0.25 \mathrm{MPa}$ and $0.30 \mathrm{MPa}$, respectively. The paint flux of the spray gun was $0.17 \mathrm{~L} / \mathrm{min}$ and the viscosity of the paint was $14.5 \mathrm{~s}$. The operational parameters were set as $23.1 \mathrm{~cm}$ of the painting width, $7.1 \mathrm{~cm}$ of interlacing distance, $6.0 \mathrm{~cm}$ of overlapping distance, $16 \mathrm{~cm}$ of the gun standoff, and $12.5 \mathrm{~cm} / \mathrm{s}$ of the sweeping speed in plane area. 
TABLE 1: Dimensionless thickness on different parts.

\begin{tabular}{lcccccc}
\hline Position & $\begin{array}{c}\text { Dimensionless } \\
\text { minimum } \\
\text { thickness }\end{array}$ & $\begin{array}{c}\text { Dimensionless } \\
\text { maximum } \\
\text { thickness }\end{array}$ & $\begin{array}{c}\text { Average } \\
\text { thickness }\end{array}$ & $\begin{array}{c}\text { Maximum positive } \\
\text { relative deviation } \\
(\%)\end{array}$ & $\begin{array}{c}\text { Maximum negative } \\
\text { relative deviation } \\
(\%)\end{array}$ & $\begin{array}{c}\text { Intolerance } \\
(\%)\end{array}$ \\
\hline Bottom of the front & 0.84 & 1.12 & 1.00 & 13.1 & 13.2 & 100 \\
Top of the front & 0.80 & 1.08 & 0.96 & 13.0 & 16.3 & 13.6 \\
Back & 0.92 & 1.16 & 0.53 & 8.9 & 100 \\
\hline
\end{tabular}

After the coating on the interior had been dry out, thickness gauge was used to measure different parts of the duct. The dimensionless thickness of the coating on different parts of the duct is shown in Table 1. Dimensionless thickness which is defined as the ratio of the measured thickness and designed thickness is used to analyze the experiment data. The dimensionless value of designed thickness is 1 . The maximum relative deviation is $16.3 \%$ at the front top of the duct. The thicknesses of all measurement points are in tolerance and satisfy the acceptance criteria proposed by the factory.

Although agreeable coating uniformity was obtained, relative deviation of the thickness distribution in the experiment was higher than that in theory, because of the following several reasons. The process of paint deposition on workpieces is influenced by some basic factors which involve spray factors including paint pressure, spray air pressure, and spray pattern pressure, paint factors including paint flow rate, viscosity, and uniformity, and workpiece factors including shape and temperature of the workpiece. But spray factors and paint factors cannot be precisely controlled during the painting operation. In addition, the adopted paint deposition model is obtained through fitting the data of experimental measurements of coating thickness distribution on planar work piece; therefore, some deviations occur when it is applied to painting curved surfaces. Lastly, the precision error of robot can cause some deviations of the actual paths and speeds off from the planned ones. Therefore, the optimal interlacing distance and overlapping distance were affected to some extent.

When painting the back of the duct, the extension of the manipulators is shorter than that painting the front, so the stiffness is higher and the dynamic accuracy is better, which makes the coating uniformity of the back part better. Therefore, the maximum thickness deviation of the front (both the top and bottom part) of the duct is higher than that of the back.

\section{Conclusion}

It is one of the important manufacture processes to paint some inner surfaces of the irregular duct of some aircrafts. Redundant robots are used to paint the duct by extending a manipulator into it. The paper presents a motion planning method of redundant manipulator for painting uniform thick coating on the interior of the irregular duct.

After analyzing the restrictions during painting the irregular duct, discontinuous peripheral painting method is presented. In order to improve the uniformity of the thick coating on the duct interior, based on the analysis of the longitudinal uniformity and transverse uniformity, the paper proposes the interlacing painting method for sweeping path planning, optimizes its parameters including overlapping distance and interlacing distance, and then presents that sweeping speeds of curved surfaces can be calculated by the ratio of transfer efficiency after the basic sweeping speed for the plane is determined.

The motion of the manipulators is controlled by restricting the distance between the links and the centerline. Joint trajectory is planned through the intertwining method, namely, minimizing the sum of the distances between the centerline of the duct and the key points on the links.

The experiment shows that the redundant manipulators can finish painting the internal surface of the S-shaped irregular duct without collision. And the paint path generated by using the interlacing method can lead to uniform coating.

Although the interlacing method is used for painting the interior of the narrow duct, it can also be practical for painting other products. And the trajectory planning method proposed by the paper can also be useful for the trajectory planning for redundant manipulators in other narrow areas.

\section{Conflict of Interests}

The authors declare that there is no conflict of interests regarding the publication of this paper.

\section{Acknowledgment}

This project is supported by National Natural Science Foundation of China (Grant no. 51475469).

\section{References}

[1] K. Liu, R. Glenn, T. J. Lawley, and F. L. Lewis, "Stewardplatform-based inlet duct painting system," in Proceedings of the IEEE International Conference on Robotics and Automation, pp. 106-113, Atlanta, Ga, USA, May 1993.

[2] Q. Lin, C. Sha, B. Zhang et al., "The development of aircraft inlet automatic spraying equipment," Manufacturing Automation, vol. 35, no. 1, pp. 92-93, 2013 (Chinese).

[3] N. A. Seegmiller, J. A. Bailiff, and R. K. Franks, "Precision robotic coating application and thickness control optimization for f-35 final finishes," SAE International Journal of Aerospace, vol. 2, no. 1, pp. 284-290, 2010.

[4] Y. Chen, J. Shao, C. Zhang, C. Fu, and K. Chen, "Redundantrobot-based painting system for variable cross-section S-shape 
pipe," in Proceedings of ASME/IFToMM International Conference on Reconfigurable Mechanisms and Robots, pp. 743-749, London, UK, June 2009.

[5] M. D. Prouty, Robotic Coating Thickness Measurement System, NAVAIR, Washington, DC, USA, 2008.

[6] H. Hyotyniemi, "Minor moves-global results: robot trajectory planning," in Proceedings of the 2nd International IEEE Conference on Tools for Artificial Intelligence, pp. 16-22, IEEE, Herndon, Va, USA, November 1990.

[7] W. Persoons and H. Van Brussel, "CAD-based robotic coating of highly curved surfaces," in Proceedings of the 24th International Symposium on Industrial Robots, pp. 611-618, Tokyo, Japan, November 1993.

[8] T. Balkan and M. A. S. Arikan, "Modeling of paint flow rate flux for circular paint sprays by using experimental paint thickness distribution," Mechanics Research Communications, vol. 26, no. 5, pp. 609-617, 1999.

[9] Y. Zhang, Y. Huang, F. Gao, and W. Wang, "New model for air spray gun of robotic spray-painting," Chinese Journal of Mechanical Engineering, vol. 42, no. 11, pp. 226-233, 2006.

[10] S.-H. Suh, I.-K. Woo, and S.-K. Noh, "Development of an automatic trajectory planning system (ATPS) for spray painting robots," in Proceedings of the IEEE International Conference on Robotics and Automation, pp. 1948-1955, Sacramento, Calif, USA, April 1991.

[11] N. Asakawa and Y. Takeuchi, "Teachless spray-paintingof sculptured surface by an industrial robot," in Proceedings of the IEEE International Conference on Robotics and Automation, vol. 3, pp. 1875-1879, Albuquerque, NM, USA, April 1997.

[12] W. Sheng, N. Xi, M. Song, Y. Chen, and P. Macneille, "Automated CAD-guided robot path planning for spray painting of compound surfaces," in Proceedings of the IEEE International Conference on Intelligent Robots and Systems, pp. 1918-1923, IEEE, Piscataway, NJ, USA, November 2000.

[13] H. Chen, W. Sheng, N. Xi, M. Song, and Y. Chen, "CAD-based automated robot trajectory planning for spray painting of freeform surfaces," Industrial Robot, vol. 29, no. 5, pp. 426-433, 2002.

[14] P. N. Atkar, A. Greenfield, D. C. Conner, H. Choset, and A. A. Rizzi, "Uniform coverage of automotive surface patches," The International Journal of Robotics Research, vol. 24, no. 11, pp. 883-898, 2005.

[15] E. S. Conkur, "Path planning using potential fields for highly redundant manipulators," Robotics and Autonomous Systems, vol. 52, no. 2-3, pp. 209-228, 2005.

[16] Y. J. Yoo, K. J. Oh, Y. J. Choi, and S. C. Won, "Obstacle avoidance for redundant manipulator without information of the joint angles," in Proceedings of the 9th Asian Control Conference (ASCC '13), pp. 1-6, Istanbul, Turkey, June 2013.

[17] M. Duguleana, F. G. Barbuceanu, A. Teirelbar, and G. Mogan, "Obstacle avoidance of redundant manipulators using neural networks based reinforcement learning," Robotics and Computer-Integrated Manufacturing, vol. 28, no. 2, pp. 132-146, 2012.

[18] A. Liegeois, "Automatic supervisory control of the configuration and behavior of multibody mechanisms," IEEE Transactions on Systems, Man and Cybernetics, vol. 7, no. 12, pp. 868-871, 1977.

[19] T. F. Chan and R. V. Dubey, "A weighted least-norm solution based scheme for avoiding joint limits for redundant joint manipulators," in Proceedings of the IEEE International Conference on Robotics and Automation, pp. 395-402, Atlanta, Ga, USA, May 1993. 


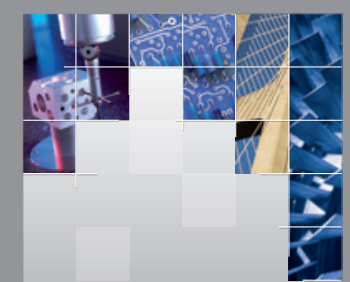

\section{Enfincering}
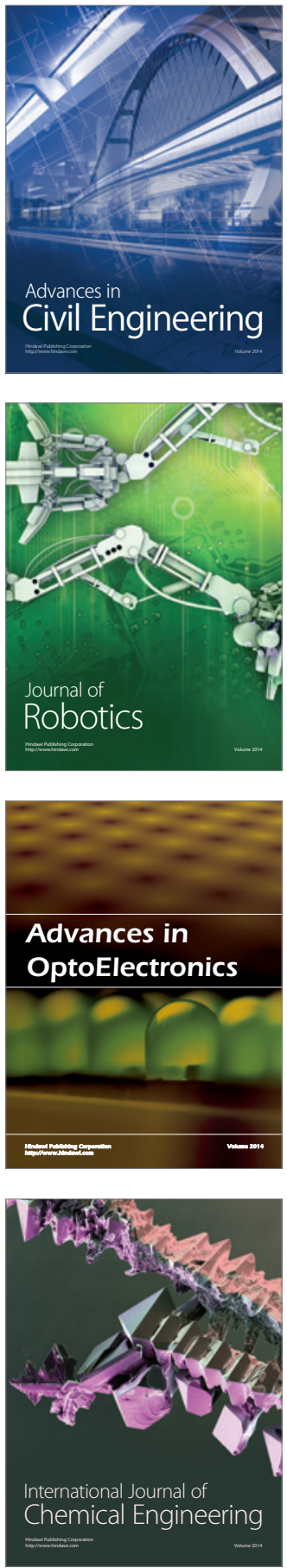

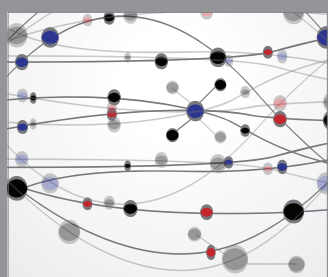

The Scientific World Journal

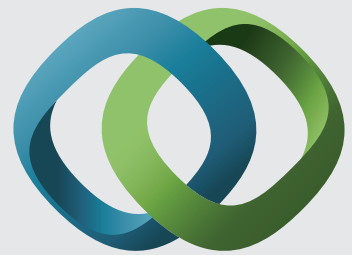

\section{Hindawi}

Submit your manuscripts at

http://www.hindawi.com
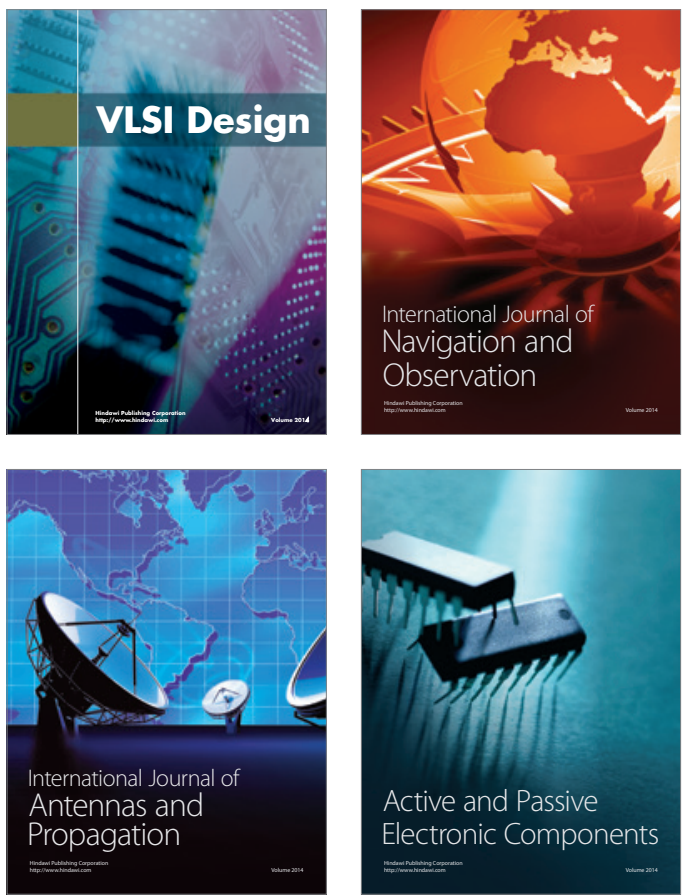
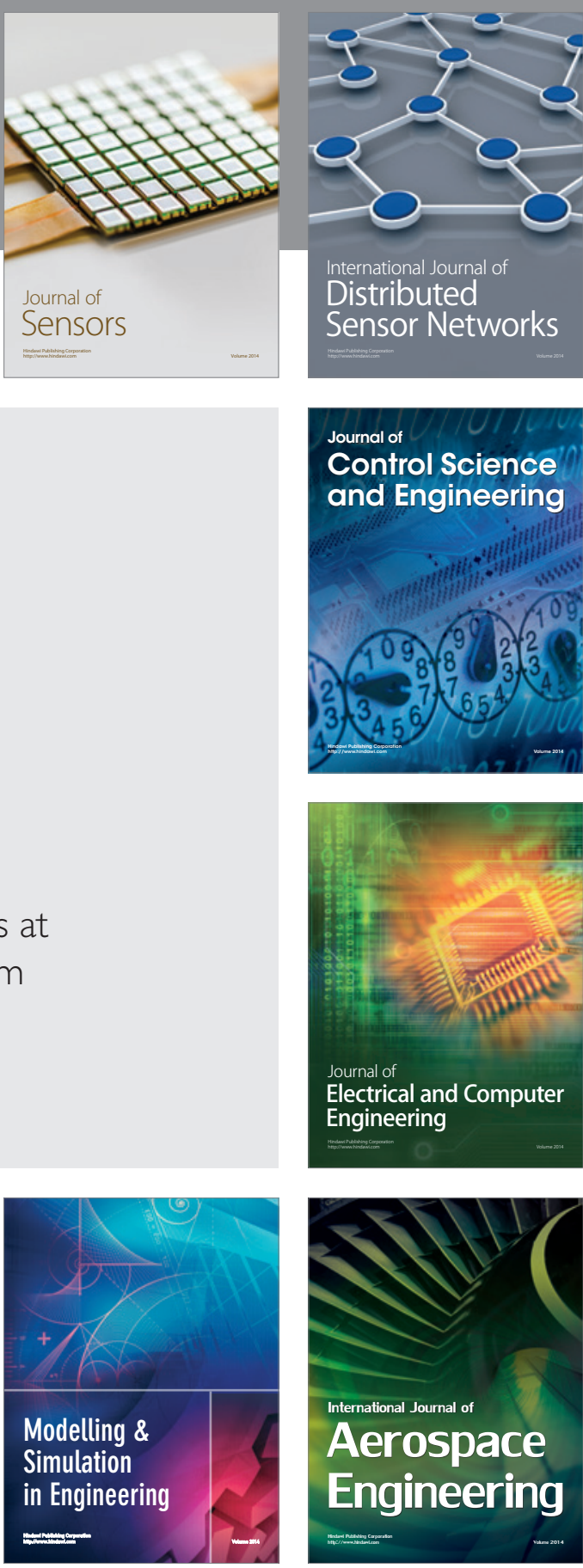

International Journal of

Distributed

Sensor Networks

Journal of

Control Science

and Engineering
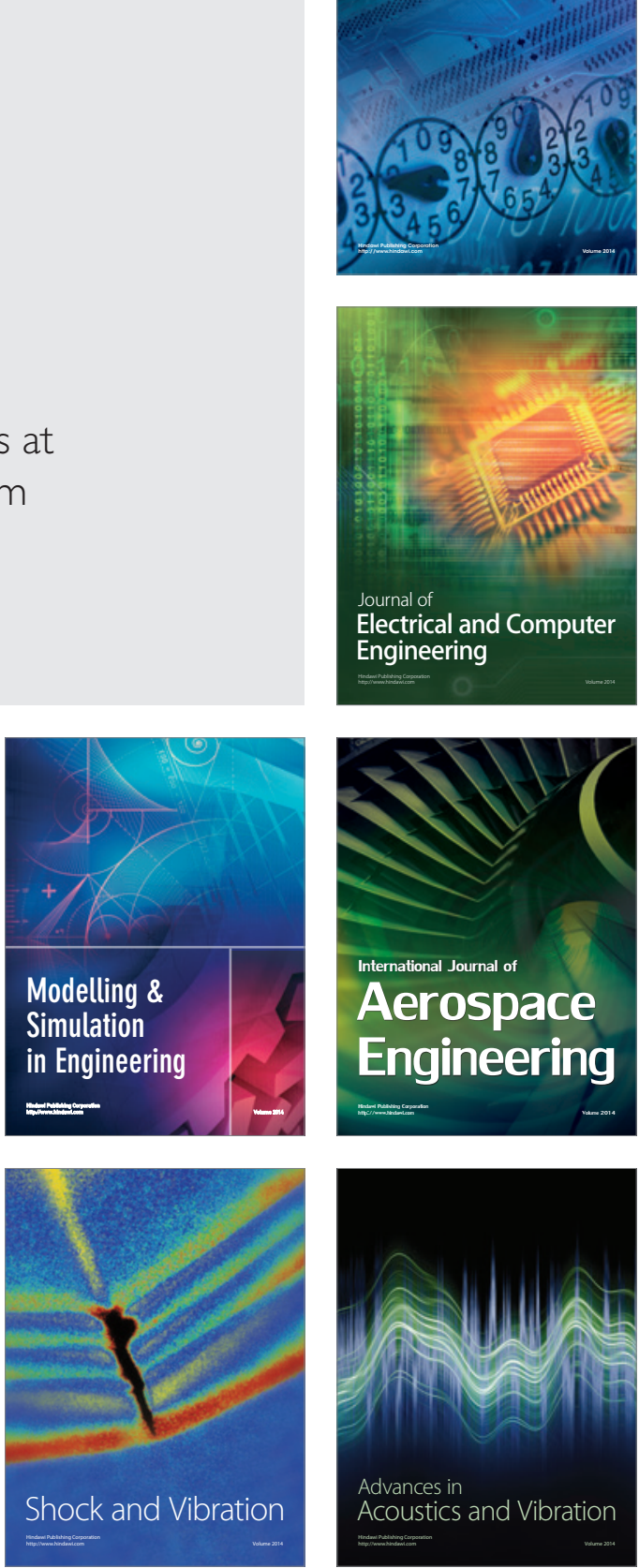